Cahiers de la recherche sur les droits

Cahiers

Fur les Droits fondamentaux

3 | 2004

Surveiller et punir / Surveiller ou punir ?

\title{
La sanction du mineur : la fuite en avant
}

\section{Christine Lazerges}

\section{OpenEdition}

Journals

Édition électronique

URL : https://journals.openedition.org/crdf/7506

DOI : $10.4000 /$ crdf. 7506

ISSN : 2264-1246

Éditeur

Presses universitaires de Caen

Édition imprimée

Date de publication : 1 octobre 2004

Pagination : 69-74

ISBN : 2-84133-221-7

ISSN : $1634-8842$

Référence électronique

Christine Lazerges, "La sanction du mineur : la fuite en avant », Cahiers de la recherche sur les droits fondamentaux [En ligne], 3 | 2004, mis en ligne le 18 décembre 2020, consulté le 14 novembre 2022 URL : http://journals.openedition.org/crdf/7506 ; DOI : https://doi.org/10.4000/crdf.7506 


\title{
La sanction du mineur : la fuite en avant ${ }^{1}$
}

\author{
Christine LAZERGES \\ Professeur à I'Université Panthéon - Sorbonne, Paris I
}

\section{Le climat de surpénalisation législative}
A. Les sanctions éducatives
B. Les centres éducatifs fermés

II. La difficile réception des textes

A. Chiffres et statistiques

B. Les centres fermés

Le si célèbre titre de l'ouvrage non moins connu de Michel Foucault ${ }^{2}$ a donné lieu à de multiples réflexions dans et hors l'université.

Surveiller et punir, surveiller ou punir? La question demeure d'une totale actualité mais pour les mineurs peut être posée un peu différemment : « socialiser et / ou punir?».

N'y a-t-il pas lieu pour les mineurs, qui aux termes de l'ordonnance du 2 février 1945 doivent prioritairement faire l'objet de mesures éducatives, de s'interroger sur la politique criminelle conduite en matière de sanctions? N'y a-t-il pas lieu de ne pas centrer son propos sur l'emprisonnement qui ne doit être que l'ultime solution et de faire cependant le constat d'une fuite en avant concernant la sanction du mineur?

Pourquoi cette terrible angoisse collective concernant les mineurs auteurs d'infractions pénales? Pourquoi cette frénésie de réformes législatives en droit des mineurs ${ }^{3}$, alors que le rapport remis au Premier Ministre en $1998^{4}$ appelait à une pause législative et préconisait expressément une pleine et entière application des textes existants. Le dernier chapitre de ce rapport portait pour titre : «Transformer radicalement les conditions d'incarcération des mineurs ». La revendication centrale des auteurs portait sur le respect de la spécificité des textes concernant les mineurs délinquants et une exigence de volontarisme dans leur application effective.

Or le mouvement de politique criminelle auquel on assiste aujourd'hui est un mouvement de « déspécialisation » du droit et de la justice des mineurs avec durcissement des sanctions encourues. Il est plus intéressant et plus significatif de se pencher sur ce mouvement en dehors de l'emprisonnement que dans le cadre de l'emprisonnement. La prison demeure dans les faits l'ultime recours, les conditions d'incarcération des mineurs n'ont pas radicalement changé, mais se sont dans de nombreuses maisons d'arrêt beaucoup améliorées.

La fuite en avant se lit dans un climat de surpénalisation législative (I) qui se heurte à une difficile réception des textes (II).

1. Ce texte emprunte pour partie à un article à paraître dans l'ouvrage Apprendre à douter: questions de droit, questions sur le droit. Études offertes à Claude Lombois, Limoges, PULIM, 2004.

2. M. Foucault, Surveiller et Punir : naissance de la prison, Paris, Gallimard, 1975.

3. Citons la loi du 9 septembre 2002 d'orientation et de programmation pour la justice dans son chapitre modifiant l'ordonnance du 2 février 1945 ; la loi du 9 mars 2004 dans ses dispositions modifiant le droit des mineurs.

4. C. Lazerges, J.-P. Balduyck, Réponses à la délinquance des mineurs, Rapport au Premier Ministre, Paris, La Documentation française, 1998. 


\section{Le climat de surpénalisation législative}

En faisant abstraction des modifications procédurales réelles et nombreuses grignotant les spécificités du procès pénal du mineur, accrues par la loi Perben II du 9 mars 2004, au seul chapitre des sanctions prévues par la loi du 9 septembre 2002, il est aisé d'illustrer le constat de surpénalisation législative.

L'ordonnance du 2 février 1945 opposait mesure éducative et sanction pénale proprement dite. Le principe se voulait être celui du prononcé d'une mesure de protection, d'assistance, de surveillance et d'éducation appropriée et l'exception, le prononcé d'une peine dès lors que pour un mineur de plus de 13 ans les circonstances et la personnalité l'exigeaient.

La modification de l'article 122-8 du Code pénal par la loi du 9 septembre 2002, en même temps qu'elle consacre la jurisprudence concernant la responsabilité du mineur, a pour objet également de durcir la répression par l'introduction d'une catégorie nouvelle de réponse: la sanction éducative, étrangement hybride.

Outre l'introduction de sanctions éducatives aux côtés des mesures éducatives et des peines, le législateur invente en réponse à une pression sociale forte, les centres éducatifs fermés, hybrides eux aussi, puisqu'ils n'ont de fermé que le nom à la différence des établissements pénitentiaires.

Les sanctions éducatives comme les centres fermés, pris pour exemple du renforcement de l'arsenal répressif à l'encontre des mineurs délinquants, sont des objets juridiques intéressants, qui bouleversent les catégories juridiques classiques. Ils constituent le noyau dur de la réponse législative actuelle à la demande sociétale de répression accrue.

\section{A. Les sanctions éducatives}

La sanction éducative présente la particularité de pouvoir être prononcée à l'égard de mineurs dès l'âge de 10 ans, lorsque la personnalité du mineur ou les circonstances l'exigent. Sans faire le choix du prononcé de peines proprement dites, le législateur affirme créer une nouvelle gamme de réponses dans laquelle les juges pourront puiser dès lors que le mineur aura atteint 10 ans.

L'ambiguïté de la sanction éducative devient indéniable dès lors que l'on se penche sur la liste des sanctions éducatives inscrites à l'article 15-1 de l'ordonnance du 2 février 1945 depuis le 9 septembre 2002. L'article 15-1 cite six sanctions éducatives cumulatives ou non et pouvant être prononcées sur décision motivée:

- confiscation d'un objet détenu ou appartenant au mineur et ayant servi à la commission de l'infraction ou qui en est le produit;

- interdiction de paraître pour une durée qui ne saurait excéder un an, dans le ou les lieux dans lesquels l'infraction a été commise et qui sont désignés par la juridiction, à l'exception des lieux dans lesquels le mineur réside habituellement;
- interdiction, pour une durée qui ne saurait excéder un an, de rencontrer ou de recevoir la ou les victimes de l'infraction désignées par la juridiction ou d'entrer en relation avec elles;

- interdiction pour une durée qui ne saurait excéder un an, de rencontrer ou de recevoir le ou les auteurs ou complices éventuels désignés par la juridiction ou d'entrer en relation avec eux.

Ces quatre premières sanctions éducatives s'analysent comme des peines complémentaires dont l'effectivité, si prononcé il y a, suppose des armées d'éducateurs. La cinquième sanction éducative présentée à l'article 15-1 de l'ordonnance est la mesure d'aide et de réparation prévue depuis 1993 à l'article 12-1 de la même ordonnance.

Voilà un législateur qui, créant une nouvelle gamme de sanctions et cherchant à l'alimenter, n'hésite pas à puiser dans des textes préexistants. La mesure d'aide ou de réparation de l'article 12-1 de l'ordonnance du 2 février 1945 est déjà une mesure éducative pouvant être retenue à tous les stades de la procédure, pour tous les mineurs y compris ceux de moins de 13 ans. Cette mesure avait déjà une double nature puisqu'elle constituait également une alternative aux poursuites. En faire en plus une sanction éducative lui confère une triple nature juridique qui signe sans doute l'engouement légitime pour une justice restauratrice par la réparation.

Il ne sert à rien cependant d'avoir fait de la mesure d'aide ou de réparation une sanction éducative, si ce n'est à ce titre d'obliger le juge à ne la prononcer que si les circonstances et la personnalité l'exigent.

La dernière des sanctions éducatives est l'obligation de suivre un stage de formation civique d'une durée qui ne peut excéder un mois, ayant pour objet de rappeler aux mineurs les obligations résultant de la loi. L'idée est excellente, la mise en ouvre difficile. À cet égard, il n'y a pas à parler de surpénalisation du mineur.

En définitive, l'intrusion des sanctions éducatives entre les mesures éducatives et les peines répond à une demande de répression. À simple examen des textes, le décalage est évident entre le discours législatif et la mise en œuvre du nouveau dispositif. Précisons que, contrairement aux mesures éducatives, les sanctions éducatives sont inscrites au bulletin numéro 1 du casier judiciaire.

Le caractère hybride de la sanction éducative est éclatant en cas de non-respect par un mineur de ladite sanction. Dans ce cas, le tribunal pour enfants pourra prononcer à son égard une mesure de placement dans l'un des établissements visés à l'article 15, c'est-à-dire sanctionner le non-respect d'une sanction éducative par une mesure éducative. Le cafouillage des catégories juridiques est total: une sanction éducative est plus lourde qu'une mesure éducative et moins lourde qu'une peine proprement dite et cependant le non-respect d'une sanction éducative est réprimée par une mesure éducative...

N’y a-t-il pas facilité et fuite en avant de la part du Parlement dans le fait d'opter pour l'illusion d'une nouvelle gamme de sanctions, alors que le système de sanction des mineurs méritait une réflexion globale? Une des pistes envisageables serait une distinction plus nette et 
claire entre les mesures éducatives et de protection prononcées en assistance éducative, sur la base des articles 375 et suivants du Code civil, et les mesures pénales qui pourraient être toutes qualifiées de sanctions éducatives. La faible lisibilité du dispositif actuel des sanctions éducatives est peu contestable; il en est de même pour les centres éducatifs fermés.

\section{B. Les centres éducatifs fermés}

Les centres éducatifs fermés, depuis la loi du 9 septembre 2002, complètent la gamme des établissements où des mineurs délinquants peuvent être placés à titre de mesures éducatives. Très judicieusement, les outils et dispositions de la justice des mineurs se diversifient, mais fallait-il pour autant revenir sur la fermeture en 1979 des derniers établissements fermés? N’est-ce pas encore une fois de la part du législateur une forme de renoncement à traiter les vrais problèmes avec pour seul gain un discours facile à médiatiser et rassurant?

L'histoire des centres fermés en France est lourde, il n'est pas question d'y revenir ${ }^{5}$, autrement que pour rappeler que si en 1979 ils furent, croyait-on, définitivement fermés, ce n'était pas par laxisme. Ces centres fermés étaient des simulacres de prison sans les garanties du statut de détenu, tous leurs défauts et effets pervers ont été dénoncés.

Pour les mineurs les plus difficiles et multirécidivistes furent créées par une loi du 14 novembre 1996 des Unités à encadrement éducatif renforcé (UEER), présentées comme le chaînon manquant entre le foyer d'hébergement classique et la prison. En 1998, sans nouvelle loi, le gouvernement décidait la poursuite de l'expérience des UEER sous le nom de Centres d'éducation renforcée (CER). Peu de temps après, le Conseil de sécurité intérieure du 27 janvier 1999 affirmait la volonté de création de 50 Centres de placement immédiat (CPI), assurant un accueil en urgence de mineurs afin qu'une observation permette par la suite une orientation vers une autre structure d'accueil. Coexistent avec ces deux types de structure des Foyers d'action éducative (FAE). Était-il nécessaire en sus de renouer avec un enfermement du mineur qui ne soit pas un enfermement dans un établissement pénitentiaire?

La plupart des candidats à l'élection présidentielle de 2002 avaient inscrit dans leur programme la renaissance des centres fermés. Il n'est pas étonnant dès lors que le législateur ait concrétisé dans la loi du 9 septembre 2002 la renaissance de structures fermées, ambiguës, puisque non confiées à la gestion de l'Administration pénitentiaire. Le Conseil constitutionnel n'émit pas même de réserves d'interprétation concernant l'instauration de centres fermés, considérant que «La dénomination de centres fermés traduit seulement le fait que la violation des obli- gations auxquelles était astreint le mineur, et notamment la sortie non autorisée du centre, est susceptible de conduire à son incarcération par révocation du contrôle judiciaire ou du sursis avec mise à l'épreuve.... Considérant, que le placement dans un centre éducatif fermé sera ordonné par l'autorité judiciaire ; que sa durée sera limitée à six mois renouvelables une fois pour le contrôle judiciaire, et à la durée de la peine d'emprisonnement pour le sursis avec mise à l'épreuve, que pour les mineurs condamnés il constitue une alternative à l'incarcération, qu'un suivi éducatif et pédagogique renforcé, adapté à la personnalité du mineur y est prévu ${ }^{6}$.

Il résulte de l'analyse de l'argumentation du Conseil constitutionnel que le placement en Centre éducatif fermé (CEF) n'est pas une incarcération, mais un placement dans un établissement éducatif au nom trompeur. Ces établissements ne seraient fermés que « juridiquement » comme l'a, à plusieurs reprises, précisé le Garde des Sceaux.

"Comment être surpris", nous dit Jean-Pierre Rosenczweig, "que des jeunes fuguent d'un établissement éducatif? Il en va ainsi depuis des décennies " ${ }^{7}$. Le manque de lisibilité de la loi du 9 septembre 2002 sur ce sujet induit un brouillage des repères et pour le mineur placé en CEF et pour le public non averti, stupéfait que l'on puisse fuguer d'un centre fermé, assimilé dans la représentation populaire à un établissement pénitentiaire pour mineurs.

La surpénalisation dans le discours s'exprime en réalité par l'intimidation du mineur placé en CEF, qui risque la prison sans être en prison.

Le sujet de l'enfermement des mineurs méritait un discours vrai, innovateur avec l'ouverture de petites unités pénitentiaires plus spécifiques pour les mineurs que ne le sont nos actuels quartiers pour mineurs dans les maisons d'arrêt, comme le proposait le récent rapport du Sénat.

À la vérité, et les centres fermés en sont un bon exemple, la surpénalisation législative des mineurs délinquants et même de leurs parents, se nourrit d'intimidation individuelle et collective. Seule une analyse de la réception de la loi du 9 septembre 2002 avec une distance suffisante dans le temps permettra de confirmer ou d'infirmer le phénomène de surpénalisation dans les faits et non plus dans la loi.

Une conclusion peut déjà être tirée de la quasiabsence de "contrôle qualité » des lois, exercé très partiellement par le Conseil constitutionnel. Avec un réel contrôle de la qualité de la loi, les catégories hybrides comme les nouvelles sanctions éducatives ou les ambiguïtés, ainsi en va-t-il du centre dit fermé et qui ne l'est pas vraiment, pourraient être gommés au bénéfice d'une recherche plus exigeante du mot juste et de la bonne qualification. Dans le champ du droit pénal, les trois fonctions de la loi gagneraient en lisibilité, qu'il s'agisse de la

5. M. Rouanet, Les Enfants du bagne, Paris, Payot, 1992; H. Gaillac, Les Maisons de correction, 1830-1945, Paris, Cujas, 1991; J.-P. Schosteck, J.-C. Carle, Délinquance des mineurs, la République en quête de respect, Rapport du Sénat, 2002, p. 184 sq.

6. Cons. Const., décision n ${ }^{\circ}$ 2002-461, DC du 29 août 2002. Commentaire C. Lazerges, D. Rousseau, Revue de droit public, 2003 , p. 1147 sq.

7. J.-P. Rosenczweig, «La chasse à l'enfant ou Thelma et Louise», Dalloz, 2003, point de vue, p. 1771. 
fonction expressive de la loi pénale, de sa fonction pédagogique ou de sa fonction répressive. Le gain en effectivité de la loi au stade de son application ne serait pas moindre. Les lois purement déclaratives de politique générale sont les plus difficiles à mettre en œuvre ${ }^{8}$.

\section{La difficile réception des textes}

Prouver la difficulté d'appliquer, s'interroger utilement sur la réception des textes, sur les freins idéologiques et politiques, suppose des études évaluatives insuffisamment entreprises et peu aisées à conduire.

Denis Szabo, dans un article sur l'évaluation des politiques criminelles, observe « qu'en ce qui concerne le choix et la construction des indicateurs, notre domaine est moins bien pourvu que les autres secteurs de la politique sociale ${ }^{9}$. De son côté, Philippe Robert note que «L'essai d'évaluation pour être sérieux, sauf à se conten- ter de définir de grandes tendances, implique le choix de terrains relativement étroits ${ }^{10}$.

Les chiffres, les statistiques sont un premier instrument d'appréciation de l'application d'un texte et, audelà, d'une politique, les travaux de terrains sont un autre mode d'évaluation de la réception de la loi. Sur la question précise de la sanction du mineur, la surpénalisation des parents et des mineurs eux-mêmes par la loi du 9 septembre 2002 est trop récente pour qu'une analyse de la jurisprudence publiée soit significative.

\section{A. Chiffres et statistiques}

Le tableau ci-dessous, extrait des chiffres clés de la justice 2003 (tableaux de bord des tribunaux pour enfants), autorise quelques observations, mais ne permet pas une analyse pour le dernier trimestre 2002 de la répartition mesures éducatives / sanctions éducatives.

Tableau 1

\begin{tabular}{|c|c|c|}
\hline Activité des juges pour enfants et des tribunaux pour enfants & 2002 & Évolution \\
\hline Mesures et sanctions définitives prises & 85509 & $-0,1$ \\
\hline Mesures rejetant la poursuite & 8441 & $+3,5$ \\
\hline Admonestations, remises à parents, dispenses de mesure ou de peine & 35678 & $-2,3$ \\
\hline Liberté surveillée, protection judiciaire, placement, réparation & 7899 & $-2,8$ \\
\hline TIG, sursis TIG & 3053 & $+6,8$ \\
\hline Amendes fermes ou avec sursis & 6760 & $-6,9$ \\
\hline Emprisonnements avec sursis simple & 10244 & $+1,1$ \\
\hline Emprisonnements avec sursis et mise à l'épreuve & 4959 & $+17,9$ \\
\hline Emprisonnements fermes & 8475 & $+2,0$ \\
\hline
\end{tabular}

Ce premier tableau doit, avant interprétation, être complété par les trois tableaux suivants :

Tableau 2

\begin{tabular}{|l|r|}
\hline Mineurs délinquants dont le juge des enfants a été saisi & 79951 \\
\hline âgés de moins de 12 ans & 3092 \\
\hline âgés de 13 à 14 ans & 15710 \\
\hline âgés de 15 à 16 ans & 38511 \\
\hline âgés de 17 ans & 22638 \\
\hline
\end{tabular}

Tableau 3

\begin{tabular}{|l|r|}
\hline Mesures présentencielles prononcées & $\mathbf{2 4 8 1 2}+16,0$ \\
\hline Enquêtes sociales, IOE, expertises & $6340+11,9$ \\
\hline Placements, liberté surveillée, réparations & $12975+15,2$ \\
\hline Contrôles judiciaires & $4073+23,8$ \\
\hline Détentions provisoires & 1424 \\
\hline
\end{tabular}

8. C. Lazerges, «De la fonction déclarative de la loi pénale», Rev. sc. crim., 2004, p. 194 sq.

9. D. Szabo, «L'évaluation des politiques criminelles: quelques réflexions préliminaires ", Rev. sc. crim., 1981, p. 1 sq.

10. P. Robert, «Évaluer la prévention », Archives de politique criminelle, n 16, 1994. 
Tableau 4

\begin{tabular}{|l|r|c|}
\hline Mineurs jugés & 63950 & $-3,8$ \\
\hline Mineurs jugés en audience de cabinet & 32792 & $-5,8$ \\
\hline Mineurs jugés en audience de TE & 31158 & $-1,5$ \\
\hline dont mineurs jugés pour crime & 443 & $-16,7$ \\
\hline
\end{tabular}

Observons la relative perméabilité auprès des juridictions pour mineurs du discours officiel répressif à l'égard des mineurs délinquants depuis déjà quelques années :

- en 2002 le juge des enfants a été saisi du même nombre de mineurs délinquants qu'en 2001, soit près de 80000 . Est-ce à dire que la délinquance des mineurs n'augmente pas, ou que les juges pour enfants ne peuvent traiter plus de 80000 dossiers par an et que le surplus serait absorbé par les mesures alternatives aux poursuites mises en œuvre par les parquets?;

- la tranche d'âge pour laquelle la délinquance augmente le plus est celle des mineurs de plus de 17 ans. Globalement la criminalité légale des mineurs de moins de 17 ans diminue contrairement à une idée reçue largement véhiculée;

- le nombre de mesures présentencielles prononcées par les juges pour enfants et les tribunaux pour enfants augmenterait sensiblement, de $+16 \%$, signe d'une prise en charge répressive plus précoce. Ainsi on note $+23,8 \%$ de contrôle judiciaire et $+21 \%$ de détentions provisoires. Ce sont là les pourcentages les plus significatifs d'une répression accrue;

- paradoxalement, le nombre de mineurs jugés diminue, que ce soit en audience de cabinet ou en audience de tribunal pour enfants pour crime ou délit;

- le renforcement des sanctions prononcées n'est significatif que concernant le travail d'intérêt général $(+6,8 \%)$ ou l'emprisonnement avec sursis et mise à l'épreuve $(+17,9 \%)$. On observe avec satisfaction une tendance lourde, dans les mesures présentencielles comme dans les sanctions définitives prises, à opter pour un accompagnement du mineur: $+15,2 \%$ pour les placements, la liberté surveillée, les mesures de réparation.

En d'autres termes, à seul examen de quelques chiffres, il serait excessif de conclure à une surpénalisation dans les faits des mineurs délinquants, mais juste de parler d'une pénalisation plus adaptée avec des réserves concernant la détention provisoire.

\section{B. Les centres fermés}

Le décalage entre le discours public et le travail de fond de la justice des mineurs est impressionnant concernant les centres fermés.

Ainsi, une analyse du discours politique, renvoyé par les médias, peut laisser croire à l'omniprésence des centres éducatifs fermés, or, au $1^{\text {er }}$ janvier 2003, sur les 327 établissements du secteur public, il n'y avait aucun CEF ouvert et sur les 775 structures d'hébergement du secteur associatif habilité, il n'y en avait que $4 \ldots$.

Sachant qu'un CEF accueille entre 8 à 10 jeunes, ce nouvel instrument issu de la loi du 9 septembre 2002 ne permet d'accueillir qu'une infime proportion des mineurs délinquants jugés par les juridictions pour mineurs. Malgré une augmentation de $100 \%$ du nombre de CEF au $1^{\text {er }}$ janvier 2004 (soit 8 contre 4), la conclusion peut être la même et prouve le danger de focaliser l'attention du public sur un instrument de la gestion de la délinquance des mineurs statistiquement ultramarginal.

Symboliquement, l'outil est moins marginal, ce que prouvent quelques enquêtes de terrains déjà conduites sur les CEF, autorisant à apprécier la réception des textes sur ce sujet.

Entretiens, auditions, enquêtes de terrain dans le cadre d'évaluations parlementaires, judiciaires ou universitaires permettent de mesurer la difficulté d'appliquer la loi.

L'ouverture de centres éducatifs fermés, dont il faut rappeler qu'ils ne s'adressent qu'aux mineurs multirécidivistes ou multiréitérants faisant l'objet d'une mesure de contrôle judiciaire ou de sursis avec mise à l'épreuve, est le plus souvent hautement conflictuelle.

La mise en place des CEF s'est heurtée d'abord à des réticences idéologiques et pédagogiques: «L'éducatif ne peut être placé sous la menace de l'incarcération », a-t-on beaucoup dit; ou encore: "Donner toute sa dimension éducative au placement est incompatible avec la menace de l'incarcération ${ }^{11}$.

Pour de nombreux éducateurs de la protection judiciaire de la jeunesse et l'immense majorité des spécialistes de l'enfance délinquante, les centres éducatifs fermés, loin de faire diminuer l'emprisonnement proprement dit des mineurs, ne feront que l'augmenter. La logique de ce type de structure est une logique d'enfermement. Les mineurs placés en CEF sont soit sous contrôle judiciaire, soit condamnés au sursis avec mise à l'épreuve avec menace d'incarcération si les obligations du contrôle judiciaire ou du sursis avec mise à l'épreuve ne sont pas respectées. Ce contexte juridique bouleverse le rapport de confiance indispensable dans le suivi éducatif.

Des entretiens avec des acteurs potentiels ou effectifs de l'encadrement des jeunes dans les CEF, il ressort une profonde réserve à l'égard de ce qui est qualifié de basculement des priorités de l'ordonnance du 2 février 1945.

Le premier frein à la réception du nouveau dispositif législatif est le discours des personnels même de la protection judiciaire de la jeunesse. Ainsi, près de 12 mois 
après le vote de la loi du 9 septembre 2002, aucun CEF public n'avait ouvert, seuls 6 CEF gérés par des associations «habilitées justice» fonctionnaient ; 2 CEF publics ont ouvert récemment.

Le second frein à la mise en place de CEF est le phénomène bien connu maintenant du NIMBY (not in my backyard). La course d'obstacles débute avec la recherche d'un bâti qui puisse être aménagé en centre éducatif fermé. Plusieurs projets se sont écroulés sous le poids des pétitions et manifestations de citoyens bien-pensants, dont des élus, favorables au dispositif et à une répression accrue des mineurs délinquants, mais pas dans leur environnement. La presse nationale et locale a rendu compte du phénomène à de nombreuses reprises ${ }^{12}$.

Loin des regards pourrait être la traduction française de l'expression NIMBY. Paradoxalement, ceux-là mêmes qui stigmatisent la délinquance des mineurs et pour qui le renforcement de la pénalisation des mineurs a été judicieusement traduit en quelques articles dans la loi du 9 septembre 2002, s'insurgent contre l'ouverture de CEF "de proximité».

Le troisième frein, non plus à l'installation, mais au bon fonctionnement d'un centre éducatif fermé est le contrôle insuffisant par la protection judiciaire de la jeunesse des établissements associatifs "habilités justice». Malgré le nombre significatif d'emplois budgétaires créés au cours des années récentes, la PJJ reste largement sousadministrée, ce qui suppose que soient remises en cause à la fois son organisation, ses procédures et ses méthodes affirme la Cour des Comptes ${ }^{13}$.
Est-ce partiellement cette insuffisance dans la tutelle des établissements privés qui explique des faux pas lourds de conséquences, ainsi en fût-il dans le premier CEF ouvert en France ${ }^{14}$ ? Ce CEF, implanté dans un ancien centre équestre, n'accueille que des jeunes filles dans un lieu où l'enfermement se résumait à quelques clôtures pour délimiter les prés et à un portail d'entrée qui n'était jamais fermé à clef ${ }^{15}$. On mesure la tromperie sur le nom et l'effet pervers sur les mineures placées qui imaginaient mal qu'une fugue aussi aisée puisse les envoyer en prison.

Centres éducatifs ou centres fermés? Telle est la question récurrente que pose la mise en œuvre de la loi du 9 septembre 2002 sur ce sujet. L'affichage politique répressif dans la loi, la fuite en avant dans les sanctions prévues par les textes, sont en total décalage avec les réalités du terrain et les besoins des jeunes en rupture.

De la loi à son application, le chemin est souvent semé d'embûches, d'autant plus incontournables que le législateur est plus attentif à l'effet recherché de son discours qu'à sa déclinaison pratique. La loi pénale serait-elle aujourd'hui d'abord l'expression d'un discours politique répondant aux attentes supposées du public ou l'expression des valeurs essentielles d'une société? La fuite en avant en matière de sanctions des mineurs est-elle la bonne solution, alors que les magistrats de la jeunesse (juges pour enfant et substituts chargés des mineurs) et l'ensemble des travailleurs sociaux impliqués dans les réponses à la délinquance des mineurs sont en attente d'une politique criminelle lisible pour tous y compris pour les mineurs?

12. Par exemple : «Le village ne veut pas céder», Paris Normandie, 12 mars 2003; ou «Saint Eulalie refuse le Star Academy de la délinquance », Sud-Ouest, 26 février 2003.

13. Cour des Comptes, La Protection judiciaire de la jeunesse, Rapport public particulier, juillet 2003.

14. CEF «EPIVIE» de Lusigny (Allier).

15. L. Dupeyron, Les Centres fermés pour mineurs délinquants de 1830 à nos jours, mémoire de DEA, Équipe de recherche sur la politique criminelle, Montpellier, 2003. 